\title{
TWO-POINT BOUNDARY VALUE PROBLEM FOR A PARTIAL DIFFERENTIAL EQUATION IN SPACES OF PERIODIC FUNCTIONS
}

\begin{abstract}
V. S. Il'kiv, Z. M. Nytrebych, P. Y. Pukach, M. I. Vovk. Two-point boundary value problem for a partial differential equation in spaces of periodic functions, Mat. Stud. 54 (2020), 79-90.
\end{abstract}

We investigate the two-point in time boundary value problem for the partial differential equations of the second-order with one spatial variable and constant coefficients. The problem is considered in in the spaces of functions which Fourier coefficients are characterized by exponential behavior on the Cartesian product of the time interval and spatial domain $\mathbb{R} / 2 \pi \mathbb{Z}$.

The correct solvability of the problem is established, the formulas for solutions are presented, the kernel is described and the smoothness of the solution is established in the spaces of functions that are periodic in one spatial variable. We have established the conditions which are close to the necessary conditions of solvability of the problem in scale of spaces of functions with exponentially increasing (or decreasing) Fourier coefficients. We also found the asymptotic estimates demonstrating the absence of the problem of small denominators, which arises of many spatial variables and makes the boundary value problem incorrect. We have established sufficient conditions of the finite-dimensionality of the kernel of the problem and found upper bounds for its dimension. The results are obtained under the condition of minimum smoothness on the right-hand sides of two-point conditions, which is close to the necessary condition.

1. Introduction. The problem of finding a solution $y(x)$ of the ordinary differential equation of the $n$th order which satisfies the $n$-point conditions

$$
y\left(x_{1}\right)=y_{1}, \ldots, y\left(x_{n}\right)=y_{n},
$$

is called the Vallée-Poussin problem ([53]), but it was considered earlier in [44, 51]. It arises naturally in studying many physical, economic, demographic and other processes. This problem was investigated in many papers, in particular, the linear case in $[1,2,35]$, the nonlinear case in $[18,20,40]$, the degenerate case in $[34,36]$ etc. Some efficient methods of constructing the approximate solutions were elaborated in [11, 52].

Recently much attention has been paid to the problems for the partial differential equations with multipoint in time conditions. The solution of such a problem is not unique unless some additional conditions with respect to spatial variables are imposed. Solvability and smoothness of the solutions are connected with the problem of small denominators. The problems of this type are the examples of the ill-posed problems in sense of Hadamard.

The metric approach to studying the multipoint problems for the partial differential equations was developed by B. Yo. Ptashnyk et al. [45, 46, 47]. The problems with the local

2010 Mathematics Subject Classification: 35B15, 35L35, 56B30.

Keywords: boundary value problem; partial differential equation; spatial variable; Fourier coefficients. doi:10.30970/ms.54.1.79-90

(C) V. S. Il'kiv, Z. M. Nytrebych, P. Y. Pukach, M. I. Vovk, 2020 
multipoint conditions were considered in [48, 50], with the nonlocal conditions in $[22,23,27$, 47] (in the complex domain in [28]), with the integral conditions in [25, 26]. The question of how to estimate the small denominators with complicated nonlinear structure is common for all types of multipoint problems ([24, 27, 47]).

The ill-posedness, in Hadamard's sense, of multipoint problems for partial differential equations is caused by the non-triviality (even by infinite-dimensionality) of the kernels. The kernels of the problems for equation of the second order in time with two-point in time conditions in the unbounded spatial domains are studied in $[3,8]$ (in the case of equations of finite order in the spatial variables) and in [41,42] (in the case of equations of infinite order).

The unique solvability of the problems for partial differential equations in unbounded domains with multipoint in time conditions in the spaces of functions of exponential growth is established in $[9,38]$. The operator method of studying the two-point problem in the strip in the Sobolev spaces is described in [12]. The two-point nonlocal problems for the weakly nonlinear differential-operator equations in the complex domain in the scales and specified scales of Sobolev spaces as well as in the scales of Dirichlet-Taylor spaces are studied by the Nash-Moser method in [29], [31] and [30], respectively. The solvability of the multipoint problems for the systems of quasilinear hyperbolic equations is proved by applying some fixed point theorems in $[4,5,7,10,49]$.

The differential-symbol method of solving the Cauchy problem for partial differential equation of finite or infinite order in spatial variables was proposed in [33, 39]. Afterwards this method was used to solve the partial differential equation of the second-order in time and of the infinite order in the spatial variables, see [43].

In the present paper we consider the two-point in time boundary value problem for the partial differential equations of the second-order with one spatial variable. The correct solvability of the problem is established, the formulas for solutions are presented, the kernel is described and the smoothness of the solution is established in the spaces of functions that are periodic in one spatial variable. We also found the asymptotic estimates demonstrating the absence of the problem of small denominators, which arises in the case of many spatial variables and makes the boundary value problem incorrect [45, 47]. The results are obtained under the condition of minimum smoothness on the right-hand sides of two-point conditions, which is close to the necessary condition.

2. Basic notations and statement of the problem. Let $\mathcal{D}=(0, h) \times \Omega$ and let $\overline{\mathcal{D}}=$ $[0, h] \times \Omega$, where $h>0, \Omega=\mathbb{R} / 2 \pi \mathbb{Z}$.

Denote by $\mathbf{W}$ the linear space of finite sums $v=v(x)=\sum_{k} v_{k} e^{i k x}$, where $v_{k}$ are the complex coefficients and $k \in \mathbb{Z}$. We call $\mathbf{W}$ a space of test functions.

The space $\mathbf{W}^{\prime}$ is dual to $\mathbf{W}$; this is the space of generalized trigonometric functions (the linear continuous functionals $V: \mathbf{W} \rightarrow \mathbb{C}$ ), that are the formal trigonometric series $V(z)=\sum_{k \in \mathbb{Z}} V_{k} e^{i k x}$ acting on the function $v \in \mathbf{W}$ by the rule $\langle V, v\rangle=\sum_{k} V_{k} \bar{v}_{k}$, where the number $\bar{v}_{k}$ is the complex conjugate of the number $v_{k}([17$, p. 59-61]).

For real numbers $q, \alpha$, positive integer $n$ and a function $\beta:[0, h] \rightarrow \mathbb{R}$ we consider the following weighted function spaces:

- the Hilbert space $\mathbf{E}_{\alpha}^{q}=\mathbf{E}_{\alpha}^{q}(\Omega)$ of periodic functions $v=v(x)=\sum_{k \in \mathbb{Z}} v_{k} e^{i k x}$ endowed with the scalar product $(v, w)_{\mathbf{E}_{\alpha}^{q}}=\sum_{k \in \mathbb{Z}}\left(1+k^{2}\right)^{q} e^{2 \alpha|k|} v_{k} \bar{w}_{k}, w=w(x)=\sum_{k \in \mathbb{Z}} w_{k} e^{i k x}$;

- the Banach space $\mathbf{E}_{\beta}^{n, q}=\mathbf{E}_{\beta}^{n, q}(\overline{\mathcal{D}})$ of functions $u=u(t, x)$ on $\overline{\mathcal{D}}$ such that their partial derivatives $\partial_{t}^{r} u(t, \cdot)$ (where $\partial_{t}=\partial / \partial t$ ) defined for $r=0,1, \ldots, n$ by the formula 
$\partial_{t}^{r} u(t, x)=\sum_{k \in \mathbb{Z}} u_{k}^{(r)}(t) e^{i k x}$ belong to the Hilbert spaces $\mathbf{E}_{\beta(t)}^{q-r}(\Omega)$ for every $t$, respectively, and are continuous in $t$ in these spaces. The squared norm of the function $u$ in the space $\mathbf{E}_{\beta}^{n, q}$ is calculated by the formula

$$
\|u\|_{\mathbf{E}_{\beta}^{n, q}}^{2}=\sum_{r=0}^{n} \max _{t \in[0, h]}\left\|\partial_{t}^{r} u(t, \cdot)\right\|_{\mathbf{E}_{\beta(t)}^{q-r}}^{2} .
$$

Note that if $\psi \in \mathbf{E}_{\alpha}^{q}$, then $\partial_{x}^{s} \psi \in \mathbf{E}_{\alpha}^{q-s}$ for all $s \in \mathbb{N}$ (where $\partial_{x}=\partial / \partial x$ ).

Consider in the domain $\mathcal{D}$ the following problem with two-point boundary conditions:

$$
\begin{gathered}
L\left(\partial_{t}, \partial_{x}\right) u \equiv \partial_{t}^{2} u-2 a\left(\partial_{x}\right) \partial_{t} u+b\left(\partial_{x}\right) u=0, \\
\left.L_{1}\left(\partial_{t}, \partial_{x}\right) u\right|_{t=0} \equiv a_{1}\left(\partial_{x}\right) \partial_{t} u+\left.b_{1}\left(\partial_{x}\right) u\right|_{t=0}=\varphi_{1}, \\
\left.L_{2}\left(\partial_{t}, \partial_{x}\right) u\right|_{t=h} \equiv a_{2}\left(\partial_{x}\right) \partial_{t} u+\left.b_{2}\left(\partial_{x}\right) u\right|_{t=h}=\varphi_{2},
\end{gathered}
$$

where

$$
\begin{aligned}
& a\left(\partial_{x}\right)=a_{0} \partial_{x}+a_{1}, \quad b\left(\partial_{x}\right)=b_{0} \partial_{x}^{2}+b_{1} \partial_{x}+b_{2}, \quad\left\{a_{0}, a_{1}, b_{0}, b_{1}, b_{2}\right\} \subset \mathbb{C} \\
& a_{\alpha}\left(\partial_{x}\right)=a_{\alpha 0} \partial_{x}+a_{\alpha 1}, b_{\alpha}\left(\partial_{x}\right)=b_{\alpha 0} \partial_{x}^{2}+b_{\alpha 1} \partial_{x}+b_{\alpha 2}, \quad\left\{a_{\alpha 0}, a_{\alpha 1}, b_{\alpha 0}, b_{\alpha 1}, b_{\alpha 2}\right\} \subset \mathbb{C}, \quad \alpha=1,2,
\end{aligned}
$$

$\varphi_{\alpha}=\varphi_{\alpha}(x)$ are the given functions defined on $\Omega$ and $u=u(t, x)$ is the unknown function.

The conditions (1) and (2) can be interpreted as fixing the spatial tensions for a onedimensional periodically oscillating body at two different moments of time $[6,13,16,37]$. These oscillation processes are described by the wave equation and some other equations $[19$, p. 25], [32, p. 77] of the form (1). Problems with similar conditions were studied also in $[14,15]$.

In view of the form of $\mathcal{D}$ and $\Omega$, we impose the conditions of $2 \pi$-periodicity with respect to the variable $x$ on the function $u$ and functions $\varphi_{1}, \varphi_{2}$, and since

$$
L\left(\partial_{t}, \partial_{x}\right) u \in \mathbf{E}_{\beta}^{0, q-2}(\overline{\mathcal{D}}),\left.\quad L_{1}\left(\partial_{t}, \partial_{x}\right) u\right|_{t=0} \in \mathbf{E}_{\beta(0)}^{q-2}(\Omega),\left.\quad L_{2}\left(\partial_{t}, \partial_{x}\right) u\right|_{t=h} \in \mathbf{E}_{\beta(h)}^{q-2}(\Omega)
$$

for any element $u \in \mathbf{E}_{\beta}^{3, q}(\overline{\mathcal{D}})$, it is naturally to use the spaces $\mathbf{E}_{\alpha}^{q}(\Omega)$ and $\mathbf{E}_{\beta}^{2, q}(\overline{\mathcal{D}})$.

Definition 1. A function $u \in \mathbf{C}^{2}\left([0, h] ; \mathbf{W}^{\prime}\right)$ is called a solution of the problem (1)-(3) if it satisfies the equation (1) on $[0, T]$, the conditions (2), (3) on the space $\mathbf{W}^{\prime}$ and belongs to the space $\mathbf{E}_{\beta}^{2, q}(\overline{\mathcal{D}})$.

The solution $u$ depends on two vectors

$$
\vec{a}=\left(a_{0}, a_{1}, a_{10}, a_{11}, a_{20}, a_{21}\right), \quad \vec{b}=\left(b_{0}, b_{1}, b_{2}, b_{10}, b_{11}, b_{12}, b_{20}, b_{21}, b_{22}\right) .
$$

The components of these vectors will be treated as the parameters of the problem which change in the bounded fixed domain. For the existence of a solution of the problem in the space $\mathbf{E}_{\beta}^{2, q}(\overline{\mathcal{D}})$ it is necessary that the right-hand sides of conditions (2), (3) have the following smoothness: $\varphi_{1} \in \mathbf{E}_{\beta(0)}^{q-2}(\Omega)$ and $\varphi_{2} \in \mathbf{E}_{\beta(h)}^{q-2}(\Omega)$.

3. Construction of solution and asymptotic estimates. We find a solution of problem (1), (2) in the form of series in the space $\mathbf{C}^{2}\left([0, h] ; \mathbf{W}^{\prime}\right)$

$$
u(t, x)=\sum_{k \in \mathbb{Z}} u_{k}(t) e^{i k x},
$$


where the coefficients $u_{k}(t)$ are the unknown functions, which will be determined by the method of separation of variables.

From the definition of solution of problem (1)-(3) it follows that the function $u_{k}=u_{k}(t)$ is the solution of the corresponding two-point problem for the ordinary differential equation

$$
\begin{aligned}
& L\left(\frac{d}{d t}, i k\right) u_{k} \equiv u_{k}^{\prime \prime}-2 a(i k) u_{k}^{\prime}+b(i k) u_{k}=0, \quad a(i k)=i k a_{0}+a_{1}, \\
& \left.L_{1}\left(\frac{d}{d t}, i k\right) u_{k}\right|_{t=0} \equiv a_{1}(i k) u_{k}^{\prime}(0)+b_{1}(i k) u_{k}(0)=\hat{\varphi}_{1 k}, \quad a_{1}(i k)=i k a_{10}+a_{11} \text {, } \\
& \left.L_{2}\left(\frac{d}{d t}, i k\right) u_{k}\right|_{t=h} \equiv a_{2}(i k) u_{k}^{\prime}(h)+b_{2}(i k) u_{k}(h)=\hat{\varphi}_{2 k}, \quad a_{2}(i k)=i k a_{20}+a_{21} \text {, }
\end{aligned}
$$

where $b(i k)=(i k)^{2} b_{0}+i k b_{1}+b_{2}$,

$$
b_{1}(i k)=(i k)^{2} b_{10}+i k b_{11}+b_{12}, \quad b_{2}(i k)=(i k)^{2} b_{20}+i k b_{21}+b_{22},
$$

and the complex numbers $\hat{\varphi}_{1 k}, \hat{\varphi}_{2 k}$ are the Fourier coefficients for the functions $\varphi_{1}, \varphi_{2}$.

The uniqueness of the solution $u_{k}$ of the problem (5)-(7) for the ordinary differential equation in the space $\mathbf{C}^{2}[0, h]$ for all $k \in \mathbb{Z}$ is necessary and sufficient condition for the uniqueness of the solution of the original problem in the space $\mathbf{C}^{2}\left([0, h] ; \mathbf{W}^{\prime}\right)$.

Denote by $\mathcal{O}_{A}(A>0)$ the closed disk $\{z:|z| \leq A\} \subset \mathbb{C}$ of some radius $A$ with center at the origin of the the complex plane and assume for convenience that $\vec{a} \in \mathcal{O}_{1}^{6}$ and $\vec{b} \in \mathcal{O}_{1}^{9}$.

In order to construct and estimate the solutions of problem (5)-(7) for $k \neq 0$ and $\alpha=1,2$, we introduce the following notations:

$$
\begin{aligned}
& \tilde{a}(k)=(i k)^{-1} a(i k), \quad a_{0}^{*}(k)=k\left(\tilde{a}(k)-a_{0}\right), \\
& \tilde{b}(k)=(i k)^{-2} b(i k), \quad b_{0}^{*}(k)=k\left(\tilde{b}(k)-b_{0}\right), \\
& \tilde{a}_{\alpha}(k)=(i k)^{-1} a_{\alpha}(i k), \quad a_{\alpha 0}^{*}(k)=k\left(\tilde{a}_{\alpha}(k)-a_{\alpha 0}\right), \\
& \tilde{b}_{\alpha}(k)=(i k)^{-2} b_{\alpha}(i k), \quad b_{\alpha 0}^{*}(k)=k\left(\tilde{b}_{\alpha}(k)-b_{\alpha 0}\right) \text {. }
\end{aligned}
$$

Then we have $\left\{a_{0}^{*}(k), a_{\alpha 0}^{*}(k)\right\} \subset \mathcal{O}_{1}$ for all $k \in \mathbb{Z}$ and

$$
\left\{\tilde{a}(k), \tilde{a}_{\alpha}(k), b_{0}^{*}(k), b_{\alpha 0}^{*}(k)\right\} \subset \mathcal{O}_{3 / 2}, \quad\left\{\tilde{b}(k), \tilde{b}_{\alpha}(k)\right\} \subset \mathcal{O}_{7 / 4}
$$

for all $|k| \geq 2$ and $\alpha=1,2$.

The solutions $\lambda_{1}(k), \lambda_{2}(k)$ of the quadratic equation

$$
\tilde{L}_{k}(\lambda) \equiv \lambda^{2}-2 \tilde{a}(k) \lambda+\tilde{b}(k)=0
$$

are determined by the formula $\lambda_{1,2}(k)=\tilde{a}(k) \pm \sqrt{D(k)}$, where $D(k)=\tilde{a}^{2}(k)-\tilde{b}(k)$ and the square root is chosen so that $\nu_{1}(k) \geq \nu_{2}(k)$, where $\nu_{1}(k)=\operatorname{Im} \lambda_{1}(k), \nu_{2}(k)=\operatorname{Im} \lambda_{2}(k)$.

If $|k| \geq 2$, then the roots of the polynomial $\tilde{L}_{k}$ satisfy [21, p. 365] the estimate

$$
\left|\lambda_{\alpha}(k)\right| \leq \max \{1+2|\tilde{a}(k)|,|\tilde{b}(k)|\} \leq 4 .
$$

Since $D(k)=D_{0}+(1 / k) D_{0}^{*}(k)$, where

$$
D_{0}=a_{0}^{2}-b_{0}, \quad D_{0}^{*}(k)=2 a_{0} a_{0}^{*}(k)+a_{0}^{* 2}(k) / k-b_{0}^{*}(k),
$$


we have $\lambda_{1,2}(k)=\lambda_{1,2 ; 0}+\frac{1}{k} \lambda_{1,2 ; 0}^{*}$, where

$$
\lambda_{1,2 ; 0}=a_{0} \pm \sqrt{D_{0}}, \quad \operatorname{Im} \lambda_{10} \geq \operatorname{Im} \lambda_{20}, \quad\left|\lambda_{1,2 ; 0}\right| \leq 1+\sqrt{2},
$$

and if $D_{0} \neq 0$, then $z=\frac{1}{k} \frac{D_{0}^{*}(k)}{D_{0}}$, where $\left.\sqrt{1+z}\right|_{z=0}=1$, and

$$
\lambda_{1,2 ; 0}^{*}=a_{0}^{*}(k) \pm \frac{D_{0}^{*}(k)}{\sqrt{D(k)}+\sqrt{D_{0}}}=a_{0}^{*}(k) \pm \frac{D_{0}^{*}(k)}{\sqrt{D_{0}}(\sqrt{1+z}+1)} .
$$

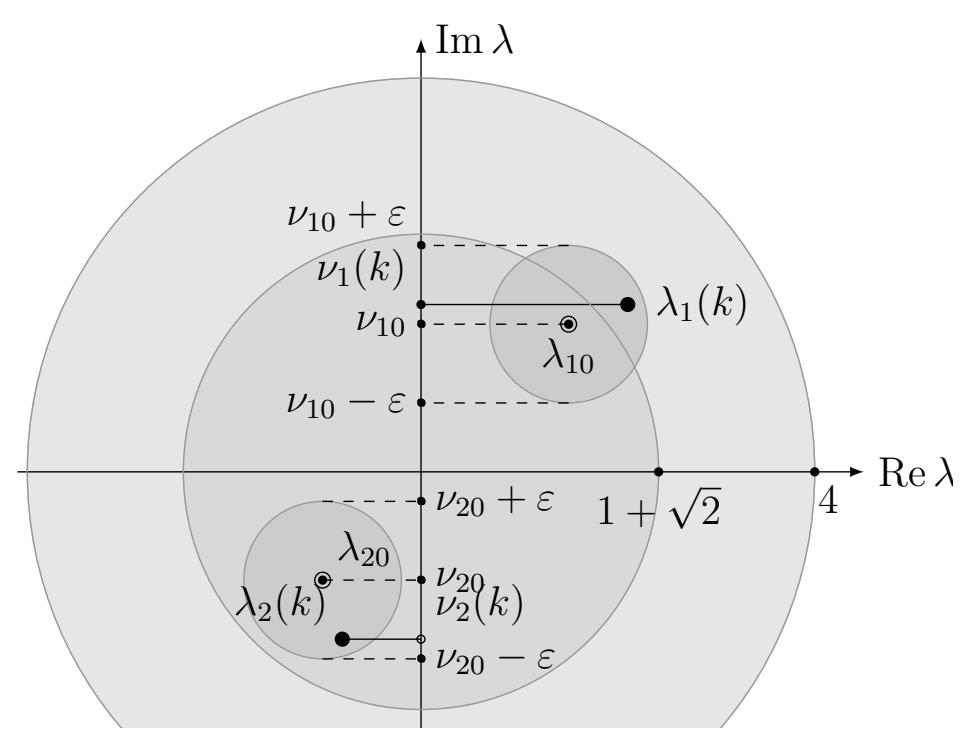

Figure 1: The location of roots of quadratic equations: $\lambda_{10}, \lambda_{20}$ are located in the middle disk, $\lambda_{1}(k)$, $\lambda_{2}(k)$ are in the big disk and if $|k| \geq K_{1}(\varepsilon)$, then $\lambda_{1}(k)$ is located on the top small disk and $\lambda_{2}(k)$ is on the bottom small disk.

It is obvious that $|\sqrt{1+z}+1| \geq 3 / 2$ for all $z \in \mathcal{O}_{3 / 4}$ and that $\left|D_{0}^{*}(k)\right| \leq 4$ for all $k \in \mathbb{Z} \backslash\{0\}$. Then

$$
\left|\lambda_{\alpha 0}^{*}\right| \leq 1+\frac{4}{\sqrt{\left|D_{0}\right|}|\sqrt{1+z}+1|} \leq 1+\frac{8 / 3}{\sqrt{\left|D_{0}\right|}} \quad \text { for } \quad|k| \geq K_{1}=\max \left\{2, \frac{16 / 3}{\left|D_{0}\right|}\right\} \geq 2 .
$$

From this estimate it follows that (see Figure 1) for any $\varepsilon>0$

$$
\left|\lambda_{\alpha}(k)-\lambda_{\alpha 0}\right|=\frac{1}{|k|}\left|\lambda_{\alpha 0}^{*}\right| \leq \varepsilon, \quad \alpha=1,2,
$$

if $|k| \geq K_{1}(\varepsilon)=\max \left\{\frac{1}{\varepsilon}\left(1+\frac{8 / 3}{\sqrt{\left|D_{0}\right|}}\right), K_{1}\right\}$.

Let $L_{\alpha k}(\lambda) \equiv L_{\alpha}(\lambda, i k)=-k^{2}\left((i k)^{-1} \tilde{a}_{\alpha}(k) \lambda+\tilde{b}_{\alpha}(k)\right)$, where $\alpha=1,2$, and let $\mathcal{K}$ be the set of integers $k$ for which the polynomial $\tilde{L}_{k}$ has a multiple root. Then $D(k) \neq 0$ and $\lambda_{1}(k) \neq \lambda_{2}(k)$ for $k \in \mathbb{Z} \backslash \mathcal{K}$.

If we take $0<\varepsilon<\left(\nu_{10}-\nu_{20}\right) / 2$, then we obtain, after using (8), that $\mathcal{K} \subset \mathcal{K}_{1}(\varepsilon)=\left\{k \in \mathbb{Z}:|k|<K_{1}(\varepsilon)\right\}, \quad\left|\lambda_{1}(k)-\lambda_{2}(k)\right| \geq \nu_{1}(k)-\nu_{2}(k) \geq \nu_{10}-\nu_{20}-2 \varepsilon>0$, 
where $\nu_{10}=\operatorname{Im} \lambda_{10}, \nu_{20}=\operatorname{Im} \lambda_{20}$.

Let $\mathcal{K}^{0}$ be the set of $k \in \mathbb{Z}$ for which $\operatorname{det} \delta_{k}=0$, where

$$
\operatorname{det} \delta_{k}=\left(a_{2}(i k) b_{1}(i k)-a_{1}(i k) b_{2}(i k)+h L_{1 k}(a(i k)) L_{2 k}(a(i k))\right) e^{h a(i k)} .
$$

For $k \in \mathcal{K} \backslash \mathcal{K}^{0}$ the solution of the problem (5)-(7) is given by the formula

$$
u_{k}(t)=\frac{\delta_{1 k}(t)}{\operatorname{det} \delta_{k}} \hat{\varphi}_{1 k}+\frac{\delta_{2 k}(t)}{\operatorname{det} \delta_{k}} \hat{\varphi}_{2 k}, \delta_{k}=\left(\begin{array}{cc}
L_{1 k}(a(i k)) & a_{1}(i k) \\
L_{2 k}(a(i k)) e^{h a(i k)} & \left(a_{2}(i k)+h L_{2 k}(a(i k))\right) e^{h a(i k))}
\end{array}\right),
$$

where $\delta_{1 k}(t)=\left(a_{2}(i k)+(h-t) L_{2 k}(a(i k)) e^{(h+t) a(i k)}, \delta_{2 k}(t)=\left(t L_{1 k}\left(a(i k)-a_{1}(i k)\right) e^{t a(i k)}\right.\right.$. For $k \in(\mathbb{Z} \backslash \mathcal{K}) \backslash \mathcal{K}^{0}$ the formula for solution has the form

$$
u_{k}(t)=\frac{\delta_{1 k}(t)}{\operatorname{det} \delta_{k}} \hat{\varphi}_{1 k}+\frac{\delta_{2 k}(t)}{\operatorname{det} \delta_{k}} \hat{\varphi}_{2 k}, \delta_{k}=\left(\begin{array}{ll}
L_{1 k}\left(i k \lambda_{1}(k)\right) & L_{1 k}\left(i k \lambda_{2}(k)\right) \\
L_{2 k}\left(i k \lambda_{1}(k)\right) e^{i k h \lambda_{1}(k)} & L_{2 k}\left(i k \lambda_{2}(k)\right) e^{i k h \lambda_{2}(k)}
\end{array}\right),
$$

where

$$
\begin{gathered}
\operatorname{det} \delta_{k}=L_{1 k}\left(i k \lambda_{1}(k)\right) L_{2 k}\left(i k \lambda_{2}(k) e^{i k h \lambda_{2}(k)}-L_{1 k}\left(i k \lambda_{2}(k)\right) L_{2 k}\left(i k \lambda_{1}(k) e^{i k h \lambda_{1}(k)},\right.\right. \\
\delta_{1 k}(t)=L_{2 k}\left(i k \lambda_{2}(k) e^{i k\left(t \lambda_{1}(k)+h \lambda_{2}(k)\right)}-L_{2 k}\left(i k \lambda_{1}(k) e^{i k\left(h \lambda_{1}(k)+t \lambda_{2}(k)\right)},\right.\right. \\
\delta_{2 k}(t)=L_{1 k}\left(i k \lambda_{1}(k)\right) e^{i k t \lambda_{2}(k)}-L_{1 k}\left(i k \lambda_{2}(k) e^{i k t \lambda_{1}(k)}\right.
\end{gathered}
$$

If $k \in \mathbb{Z} \backslash \mathcal{K}$ and is such that $k R_{1}(k) R_{2}(k) \tilde{b}(k) \neq 0$, where for $\alpha=1,2$,

$$
R_{\alpha}(k)=L_{\alpha k}\left(i k \lambda_{1}(k)\right) L_{\alpha k}\left(i k \lambda_{2}(k)\right)=k^{4}\left(\tilde{a}_{\alpha}^{2}(k) \tilde{b}(k)+2 \tilde{a}(k) \tilde{a}_{\alpha}(k) \tilde{b}_{\alpha}(k)+\tilde{b}_{\alpha}^{2}(k)\right)
$$

is the resultant of the polynomials $L_{\alpha k}$ and $L(\cdot, i k)$, then in the case $k>0$ the formula for solution (10) and its derivatives has the following form:

$$
u_{k}^{(j)}(t)=\frac{e^{i k \lambda_{1}(k) t}}{\lambda_{1}^{-j}(k)} \frac{L_{1 k}\left(i k \lambda_{2}(k)\right)}{R_{1}(k)} \frac{\Delta_{1 k}^{j+}(t)}{\Delta_{k}^{+}} \frac{\hat{\varphi}_{1 k}}{(i k)^{-j}}+\frac{e^{-i k \lambda_{2}(k)(h-t)}}{\lambda_{2}^{-j}(k)} \frac{L_{2 k}\left(i k \lambda_{1}(k)\right)}{R_{2}(k)} \frac{\Delta_{2 k}^{j+}(t)}{\Delta_{k}^{+}} \frac{\hat{\varphi}_{2 k}}{(i k)^{-j}},
$$

where

$$
\begin{gathered}
\Delta_{1 k}^{j+}(t)=1-\frac{L_{2 k}^{2}\left(i k \lambda_{1}(k)\right)}{R_{2}(k)} \frac{\lambda_{2}^{2 j}(k)}{\tilde{b}^{j}(k)} e^{i 2 k D(k)(h-t)}, \quad j=1,2, \\
\Delta_{2 k}^{j+}(t)=1-\frac{L_{1 k}^{2}\left(i k \lambda_{2}(k)\right)}{R_{1}(k)} \frac{\lambda_{1}^{2 j}(k)}{\tilde{b}^{j}(k)} e^{i 2 k D(k) t}, \quad j=1,2, \\
\Delta_{k}^{+}=1-\frac{L_{1 k}^{2}\left(i k \lambda_{2}(k)\right) L_{2 k}^{2}\left(i k \lambda_{1}(k)\right)}{R_{1}(k) R_{2}(k)} e^{i 2 k D(k) h} \neq 0
\end{gathered}
$$

and in the case $k<0$

$$
u_{k}^{(j)}(t)=\frac{e^{i k \lambda_{2}(k) t}}{\lambda_{2}^{-j}(k)} \frac{L_{1 k}\left(i k \lambda_{1}(k)\right)}{R_{1}(k)} \frac{\Delta_{1 k}^{j-}(t)}{\Delta_{k}^{-}} \frac{\hat{\varphi}_{1 k}}{(i k)^{-j}}+\frac{e^{-i k \lambda_{1}(k)(h-t)}}{\lambda_{1}^{-j}(k)} \frac{L_{2 k}\left(i k \lambda_{2}(k)\right)}{R_{2}(k)} \frac{\Delta_{2 k}^{j-}(t)}{\Delta_{k}^{-}} \frac{\hat{\varphi}_{2 k}}{(i k)^{-j}},
$$

where

$$
\Delta_{1 k}^{j-}(t)=1-\frac{L_{2 k}^{2}\left(i k \lambda_{2}(k)\right)}{R_{2}(k)} \frac{\lambda_{1}^{2 j}(k)}{\tilde{b}^{j}(k)} e^{-i 2 k D(k)(h-t)}, \quad j=1,2,
$$




$$
\begin{aligned}
\Delta_{2 k}^{j-}(t) & =1-\frac{L_{1 k}^{2}\left(i k \lambda_{1}(k)\right)}{R_{1}(k)} \frac{\lambda_{2}^{2 j}(k)}{\tilde{b}^{j}(k)} e^{-i 2 k D(k) t}, \quad j=1,2, \\
\Delta_{k}^{-} & =1-\frac{L_{1 k}^{2}\left(i k \lambda_{1}(k)\right) L_{2 k}^{2}\left(i k \lambda_{2}(k)\right)}{R_{1}(k) R_{2}(k)} e^{-i 2 k D(k) h} \neq 0 .
\end{aligned}
$$

Let us estimate derivatives of the solution $u_{k}(t)$ of the problem (5)-(7). We use the following relations:

$L_{\alpha k}\left(i k \lambda_{\beta}(k)\right)=-k^{2}\left(L_{\alpha \beta 0}+(1 / k) L_{\alpha \beta 0}^{*}\right), \quad R_{\alpha}(k)=k^{4}\left(R_{\alpha 0}+(1 / k) R_{\alpha 0}^{*}\right), \quad\{\alpha, \beta\} \subset\{1,2\}$,

where

$$
\begin{aligned}
& L_{\alpha \beta 0}=a_{\alpha 0} \lambda_{\beta 0}+b_{\alpha 0}, L_{\alpha \beta 0}^{*}=a_{\alpha 0}^{*} \lambda_{\beta 0}+a_{\alpha 0} \lambda_{\beta 0}^{*}+(1 / k) a_{\alpha 0}^{*} \lambda_{\beta 0}^{*}, R_{\alpha 0}=a_{\alpha 0}^{2} b_{0}+2 a_{0} a_{\alpha 0} b_{\alpha 0}+b_{\alpha 0}^{2}, \\
& R_{\alpha 0}^{*}=\left(a_{\alpha 0}+\tilde{a}(k)\right) a_{\alpha 0}^{*} b_{0}+\tilde{a}^{2}(k) b_{0}^{*}+2\left(a_{0}^{*} a_{\alpha 0}+\tilde{a}(k) a_{\alpha 0}^{*}\right) b_{\alpha 0}+\left(2 \tilde{a}(k) \tilde{a}_{\alpha}(k)+b_{\alpha 0}+\tilde{b}(k)\right) b_{\alpha 0}^{*},
\end{aligned}
$$

from which it follows that $\left|L_{\alpha k}\left(i k \lambda_{\beta}(k)\right)\right| \leq 8 k^{2}$ for $|k| \geq 2,\left|R_{\alpha 0}^{*}\right|<21$, and also that $\left|R_{\alpha}(k)\right| \geq k^{4}\left|R_{\alpha 0}\right| / 2$ for $|k| \geq 42$.

Using the obtained estimates and the inequality

$$
\nu_{20}-\varepsilon<\nu_{2}(k)<\nu_{20}+\varepsilon<\nu_{10}-\varepsilon<\nu_{1}(k)<\nu_{10}+\varepsilon, \quad|k| \geq K_{1}(\varepsilon),
$$

for an arbitrary $0<\varepsilon<\left(\nu_{10}-\nu_{20}\right) / 2$ and letting $K_{2}(\varepsilon)=\max \left\{K_{1}(\varepsilon), 42\right\}$, we find that

$$
\begin{gathered}
\left|u_{k}^{(j)}(t)\right| \leq \frac{4^{j+2}}{k^{2-j}}\left(e^{-k\left(\nu_{10}-\varepsilon\right) t} \frac{\left|\Delta_{1 k}^{j+}(t) \hat{\varphi}_{1 k}\right|}{\left|\Delta_{k}^{+} R_{10}\right|}+e^{k\left(\nu_{20}+\varepsilon\right)(h-t)} \frac{\left|\Delta_{2 k}^{j+}(t) \hat{\varphi}_{2 k}\right|}{\left|\Delta_{k}^{+} R_{20}\right|}\right) \quad \text { for } \quad k \geq K_{2}(\varepsilon), \quad(13) \\
\left|u_{k}^{(j)}(t)\right| \leq \frac{4^{j+2}}{k^{2-j}}\left(e^{-k\left(\nu_{20}+\varepsilon\right) t} \frac{\left|\Delta_{1 k}^{j-}(t) \hat{\varphi}_{1 k}\right|}{\left|\Delta_{k}^{-} R_{10}\right|}+e^{k\left(\nu_{10}-\varepsilon\right)(h-t)} \frac{\left|\Delta_{2 k}^{j-}(t) \hat{\varphi}_{2 k}\right|}{\left|\Delta_{k}^{-} R_{20}\right|}\right) \quad \text { for } \quad k \leq-K_{2}(\varepsilon) .
\end{gathered}
$$

Basing on the estimates $\left|\Delta_{1 k}^{ \pm}(t)\right| \leq 1+2^{7+5 j} /\left|R_{20} b_{0}^{j}\right|,\left|\Delta_{2 k}^{ \pm}(t)\right| \leq 1+2^{7+5 j} /\left|R_{10} b_{0}^{j}\right|$, we can rewrite formulas (13) and (14) for the case

$$
|k| \geq K_{3}(\varepsilon)=\max \left\{K_{2}(\varepsilon), 8 \ln 2 /\left(\nu_{10}-\nu_{20}-2 \varepsilon\right)\right\} .
$$

Letting $C_{1} \equiv 2^{22} /\left|R_{10} R_{20}\right|$, we get

$$
\begin{aligned}
& \left|u_{k}^{(j)}(t)\right|^{2} \leq \frac{2 C_{1}^{2}}{\left|b_{0}\right|^{2 j}}\left(1+k^{2}\right)^{j-2}\left(e^{2\left(\varepsilon-\nu_{10}\right) t|k|}\left|\hat{\varphi}_{1 k}\right|^{2}+e^{2\left(\nu_{20}+\varepsilon\right)(h-t)|k|}\left|\hat{\varphi}_{2 k}\right|^{2}\right) \quad \text { for } \quad k \geq K_{3}(\varepsilon), \\
& \left|u_{k}^{(j)}(t)\right|^{2} \leq \frac{2 C_{1}^{2}}{\left|b_{0}\right|^{2 j}}\left(1+k^{2}\right)^{j-2}\left(e^{2\left(\nu_{20}+\varepsilon\right) t|k|}\left|\hat{\varphi}_{1 k}\right|^{2}+e^{2\left(\varepsilon-\nu_{10}\right)(h-t)|k|}\left|\hat{\varphi}_{2 k}\right|^{2}\right) \quad \text { for } k \leq-K_{3}(\varepsilon) .
\end{aligned}
$$

4. Solvability of the problem. The last step in solving the problem (1)-(3) is to determine the solutions of the problem $(5)-(7)$ for $k \in \mathcal{K}^{0}$, i.e., in the case of the degenerate matrix $\delta_{k}$ which rank is either zero $\left(\delta_{k}=0, k \in \mathcal{K}^{00}\right)$, or one $\left(k \in \mathcal{K}^{10}\right)$. In this case the solution does not exist or is not unique. To describe all solutions, we let $\delta_{k}=\left(\begin{array}{c}\Gamma_{11}(k) \Gamma_{12}(k) \\ \Gamma_{21}(k) \Gamma_{22}(k)\end{array}\right)$, and denote 
by $G_{i j}(k)$ the conjugate of the complex number $\Gamma_{i j}(k)$, and by $\left(\gamma_{1 k}, \gamma_{2 k}\right)$ an arbitrary vector in the space $\mathbb{C}^{2}$.

If $\delta_{k}=0\left(k \in \mathcal{K}^{00}\right)$, then the necessary and sufficient condition for the existence of solution is that

$$
\hat{\varphi}_{1 k}=\hat{\varphi}_{2 k}=0 .
$$

The space of solutions (the kernel of problem (5)-(7)), in this case, is two-dimensional and consists of all the functions

$$
\begin{gathered}
u_{k}(t)=e^{t a(i k)}\left(\gamma_{1 k}+t \gamma_{2 k}\right), \quad k \in \mathcal{K}, \\
u_{k}(t)=e^{i k t \lambda_{1}(k)} \gamma_{1 k}+e^{i k t \lambda_{2}(k)} \gamma_{2 k}, \quad k \notin \mathcal{K} .
\end{gathered}
$$

If $\delta_{k}$ has rank one $\left(k \in \mathcal{K}^{10}\right)$, then it also has nonzero row and nonzero column. The necessary and sufficient condition for the solvability is the following condition of proportionality:

$$
\Gamma_{21}(k) \hat{\varphi}_{1 k}=\Gamma_{11}(k) \hat{\varphi}_{2 k}, \quad \Gamma_{22}(k) \hat{\varphi}_{1 k}=\Gamma_{12}(k) \hat{\varphi}_{2 k} .
$$

The solutions of the problem in the case $S_{1}(k) \equiv\left|\Gamma_{11}(k)\right|^{2}+\left|\Gamma_{12}(k)\right|^{2}>0$ have the form:

$$
u_{k}(t)=e^{t a(i k)}\left(\begin{array}{ll}
1 & t
\end{array}\right)\left(\left(\begin{array}{c}
G_{11}(k) \\
G_{12}(k)
\end{array}\right) \frac{\hat{\varphi}_{1 k}}{S_{1}(k)}+\left(\begin{array}{cc}
\left|\Gamma_{12}(k)\right|^{2} & -G_{11}(k) \Gamma_{12}(k) \\
-\Gamma_{11}(k) G_{12}(k) & \left|\Gamma_{11}(k)\right|^{2}
\end{array}\right)\left(\begin{array}{c}
\gamma_{1 k} \\
\gamma_{2 k}
\end{array}\right)\right)
$$

for $k \in \mathcal{K}$. If $S_{1}(k)=0$, then $S_{2}(k) \equiv\left|\Gamma_{21}(k)\right|^{2}+\left|\Gamma_{22}(k)\right|^{2}>0$ and

$$
u_{k}(t)=e^{t a(i k)}\left(\begin{array}{ll}
1 & t
\end{array}\right)\left(\left(\begin{array}{l}
G_{21}(k) \\
G_{22}(k)
\end{array}\right) \frac{\hat{\varphi}_{2 k}}{S_{2}(k)}+\left(\begin{array}{cc}
\left|\Gamma_{22}(k)\right|^{2} & -G_{21}(k) \Gamma_{22}(k) \\
-\Gamma_{21}(k) G_{22}(k) & \left|\Gamma_{21}(k)\right|^{2}
\end{array}\right)\left(\begin{array}{l}
\gamma_{1 k} \\
\gamma_{2 k}
\end{array}\right)\right)
$$

for $k \in \mathcal{K}$.

In the case $k \in \mathcal{K}^{0} \backslash \mathcal{K}$, we have the analogical formulas:

$$
\begin{aligned}
& u_{k}(t)=\left(\begin{array}{ll}
e^{i k t \lambda_{1}(k)} & e^{i k t \lambda_{2}(k)}
\end{array}\right)\left(\left(\begin{array}{l}
G_{11}(k) \\
G_{12}(k)
\end{array}\right) \frac{\hat{\varphi}_{1 k}}{S_{1}(k)}+\left(\begin{array}{cc}
\left|\Gamma_{12}(k)\right|^{2} & -G_{11}(k) \Gamma_{12}(k) \\
-\Gamma_{11}(k) G_{12}(k) & \left|\Gamma_{11}(k)\right|^{2}
\end{array}\right)\left(\begin{array}{l}
\gamma_{1 k} \\
\gamma_{2 k}
\end{array}\right)\right), \\
& u_{k}(t)=\left(\begin{array}{ll}
e^{i k t \lambda_{1}(k)} & e^{i k t \lambda_{2}(k)}
\end{array}\right)\left(\left(\begin{array}{l}
G_{21}(k) \\
G_{22}(k)
\end{array}\right) \frac{\hat{\varphi}_{2 k}}{S_{2}(k)}+\left(\begin{array}{cc}
\left|\Gamma_{22}(k)\right|^{2} & -G_{21}(k) \Gamma_{22}(k) \\
-\Gamma_{21}(k) G_{22}(k) & \left|\Gamma_{21}(k)\right|^{2}
\end{array}\right)\left(\begin{array}{c}
\gamma_{1 k} \\
\gamma_{2 k}
\end{array}\right)\right) .
\end{aligned}
$$

Theorem 1 (Existence of formal solution). Let the functions $\varphi_{1}$ and $\varphi_{2}$ belong to the space $\mathbf{W}^{\prime}$ and satisfy the conditions (20) for $k \in \mathcal{K}^{0}$ as well as the condition (17) for $k \in \mathcal{K}^{00}$, then a solution of the problem (1)-(3) exists in the space $\mathbf{C}^{2}\left([0, h] ; \mathbf{W}^{\prime}\right)$. The solution is unique, if $\mathcal{K}^{0}=\varnothing$.

Proof. The solution has the form (4) with the coefficients $u_{k}$ calculated by formulas (9) and (10), if $k \in \mathbb{Z} \backslash \mathcal{K}^{0}$, and by formulas (21)-(24), if $k \in \mathcal{K}^{0}$.

Assume that $\mathcal{K}^{0} \neq \varnothing$. Then for any $k \in \mathcal{K}^{0}$ the corresponding homogeneous problem (1)-(3) has the nontrivial solution, that belongs to the set

$$
\left\{e^{t a(i k)}\left(\left|\Gamma_{12}(k)\right|^{2}-t G_{11}(k) \Gamma_{12}(k)\right), \quad e^{t a(i k)}\left(\left|\Gamma_{22}(k)\right|^{2}-t G_{21}(k) \Gamma_{22}(k)\right)\right\},
$$

if $k \in \mathcal{K}$, or belongs to the set

$$
\left\{e^{i k t \lambda_{1}(k)}\left(\left|\Gamma_{12}(k)\right|^{2}-e^{i k t \lambda_{2}(k)} G_{11}(k) \Gamma_{12}(k)\right), \quad e^{i k t \lambda_{1}(k)}\left|\Gamma_{22}(k)\right|^{2}-e^{i k t \lambda_{1}(k)} G_{21}(k) \Gamma_{22}(k)\right\},
$$

if $k \notin \mathcal{K}$. 
Theorem 2 (Existence of solution). Let vectors $\vec{a}$ and $\vec{b}$ of the problem (1)-(3) satisfy the condition $b_{0}\left(D_{0}-\alpha\right) R_{10} R_{20} \neq 0$ for all $\alpha \geq 0$. Then the problem (1)-(3) can have only finite-dimensional kernel whose dimension is not greater than $4 K_{3}(\varepsilon)-2$ for any $0<\varepsilon<$ $\left(\nu_{10}-\nu_{20}\right) / 2$. If the functions $\varphi_{1}, \varphi_{2}$ satisfy the conditions of Theorem 1 and belong to the space $\mathbf{E}_{h \nu_{\varepsilon}^{*} / 2}^{q-2}(\Omega)$, then the solution $u=u_{\bullet}+u_{+}+u_{-}$of the problem (1)-(3) exists in the space $\mathbf{E}_{\theta}^{2, q}(\overline{\mathcal{D}})$, where

$$
u_{\bullet}=\sum_{k=1-K_{3}(\varepsilon)}^{K_{3}(\varepsilon)-1} u_{k}(t) e^{i k x}, \quad u_{+}=\sum_{k=K_{3}(\varepsilon)}^{\infty} u_{k}(t) e^{i k x}, \quad u_{-}=\sum_{k=K_{3}(\varepsilon)}^{\infty} u_{-k}(t) e^{-i k x},
$$

$\theta(t)=\min \left\{(t-h / 2) \nu_{\varepsilon}^{*},(h / 2-t) \nu_{\varepsilon}^{*}\right\}, \nu_{\varepsilon}^{*}=\max \left\{\varepsilon-\nu_{10}, \nu_{20}+\varepsilon\right\}$, and the inequality

$$
\left\|u_{+}+u_{-}\right\|_{\mathbf{E}_{\theta}^{2, q}}^{2} \leq 2\left(1+\left|b_{0}\right|^{-2}+\left|b_{0}\right|^{-4}\right) C_{1}^{2}\left(\left\|\varphi_{1}\right\|_{\mathbf{E}_{h \nu_{\varepsilon}^{*} / 2}^{q-2}}^{2}+\left\|\varphi_{2}\right\|_{\mathbf{E}_{h \nu_{\varepsilon}^{*} / 2}^{q-2}}^{2}\right)
$$

holds.

Proof. From the conditions of the present theorem it follows that the solution of the problem (5)-(7) is unique when $|k| \geq K_{3}(\varepsilon)$. Hence, the kernel of the problem (1)-(3) is generated by the $\left(2 K_{3}(\varepsilon)-1\right)$-element set $\left\{k \in \mathbb{Z}:|k|<K_{3}(\varepsilon)\right\}$ each element of which is associated with two or one linear independent elements of kernel.

In order to prove the inequality (26), we use the formula

$$
\left|u_{k}^{(j)}(t)\right|^{2} \leq \frac{2 C_{1}^{2}}{\left|b_{0}\right|^{2 j}}\left(1+k^{2}\right)^{j-2}\left(e^{2 \nu_{\varepsilon}^{*} t|k|}\left|\hat{\varphi}_{1 k}\right|^{2}+e^{2 \nu_{\varepsilon}^{*}(h-t)|k|}\left|\hat{\varphi}_{2 k}\right|^{2}\right), \quad|k| \geq K_{3}(\varepsilon),
$$

which is a consequence of (15), (16). Passing to the norms, we obtain the inequality

$$
\left\|u_{+}+u_{-}\right\|_{\mathbf{E}_{\theta}^{2, q}}^{2} \leq 2 B_{0} C_{1}^{2} \sum_{|k| \geq K_{3}(\varepsilon)}\left(1+k^{2}\right)^{q-2}\left(e^{2\left(\theta(t)+\nu_{\varepsilon}^{*} t\right)|k|}\left|\hat{\varphi}_{1 k}\right|^{2}+e^{2\left(\theta(t)+\nu_{\varepsilon}^{*}(h-t)\right)|k|}\left|\hat{\varphi}_{2 k}\right|^{2}\right)
$$

(where $B_{0}=1+\left|b_{0}\right|^{-2}+\left|b_{0}\right|^{-4}$ ) which proves $(26)$.

The condition $b_{0}\left(D_{0}-\alpha\right) R_{10} R_{20} \neq 0$ of Theorem 2 means that the quadratic trinomial $\lambda^{2}-2 a_{0} \lambda+b_{0}$ does not have zero roots; its roots are not located on horizontal line and are not the roots of the quadratic trinomial $\left(a_{10} \lambda+b_{10}\right)\left(a_{20} \lambda+b_{20}\right)$.

The following theorems demonstrate the increased smoothness of some parts of the solution.

Theorem 3 (Increase of the solution smoothness). Let the conditions of Theorem 2 hold, and let $\varphi_{2}$ be a trigonometric polynomial. Then a solution of the problem (1)-(3) exists and belongs to the space $\mathbf{E}_{(h / 2-t) \nu_{\varepsilon}^{*}}^{2, q}(\overline{\mathcal{D}})$. If $\varphi_{1}$ is a trigonometric polynomial, then a solution of the problem (1)-(3) also exists and belongs to the space $\mathbf{E}_{(t-h / 2) \nu_{\varepsilon}^{*}}^{2, q}(\overline{\mathcal{D}})$.

Proof. According to Theorem 1, the solution of the problem (1)-(3) exists. In particular, $u=u_{\bullet}+u_{*}+u_{* *}$, where $u=u_{\bullet}$ and $u_{* *}$ are polynomials, and $u_{*}$ does not depend on the function $\varphi_{2}$, i.e., $u_{*}=\sum_{|k| \geq K_{3}(\varepsilon)} \frac{\delta_{1 k}(t)}{\operatorname{det} \delta_{k}} \hat{\varphi}_{1 k} e^{i k x}$.

The estimate for $u_{*}$ is a consequence of the formula (27), where $\varphi_{2}=0$, namely

$$
\left\|u_{*}\right\|_{\mathbf{E}_{(h / 2-t) \nu_{\varepsilon}^{*}}^{2, q}}^{2} \leq 2 B_{0} C_{1}^{2} \sum_{|k| \geq K_{3}(\varepsilon)}\left(1+k^{2}\right)^{q-2} e^{2\left((h / 2-t) \nu_{\varepsilon}^{*}+\nu_{\varepsilon}^{*} t\right)|k|}\left|\hat{\varphi}_{1 k}\right|^{2}=
$$




$$
=2 B_{0} C_{1}^{2} \sum_{|k| \geq K_{3}(\varepsilon)}\left(1+k^{2}\right)^{q-2} e^{h \nu_{\varepsilon}^{*}|k|}\left|\hat{\varphi}_{1 k}\right|^{2} \leq 2 B_{0} C_{1}^{2}\left\|\varphi_{1}\right\|_{\mathbf{E}_{h \nu_{\varepsilon}^{*} / 2}^{q-2}} .
$$

Similarly, if $\varphi_{1}$ is polynomial, then $u=u_{\bullet}+u_{*}+u_{* *}$, where $u=u_{\bullet}$ and $u_{*}$ are polynomials, and the estimate $\left\|u_{* *}\right\|_{\mathbf{E}_{(t-h / 2) \nu_{\varepsilon}^{*}}^{2, q}} \leq 2 B_{0} C_{1}^{2}\left\|\varphi_{2}\right\|_{\mathbf{E}_{h \nu_{\varepsilon}^{*} / 2}^{q-2}}$ is obtained also from (27), if $\varphi_{1}=0$.

It is obvious that the spaces $\mathbf{E}_{ \pm(t-h / 2) \nu_{\varepsilon}^{*}}^{2, q}(\overline{\mathcal{D}})$ are subspaces of the space $\mathbf{E}_{\theta(t) \nu_{\varepsilon}^{*}}^{2, q}(\overline{\mathcal{D}})$, which is used in Theorem 2. Hence the smoothness of the solution increases.

Let us separate the right-hand sides of the conditions (2), (3) into three parts $\varphi_{j}=$ $\varphi_{j}^{\bullet}+\varphi_{j}^{+}+\varphi_{j}^{-}$, where

$$
\varphi_{j}^{\bullet}=\sum_{k=1-K_{3}(\varepsilon)}^{K_{3}(\varepsilon)-1} \hat{\varphi}_{j k} e^{i k x}, \quad \varphi_{j}^{+}=\sum_{k=K_{3}(\varepsilon)}^{\infty} \hat{\varphi}_{j k} e^{i k x}, \quad \varphi_{j}^{-}=\sum_{k=K_{3}(\varepsilon)}^{\infty} \hat{\varphi}_{j,-k} e^{-i k x}, \quad j=1,2 .
$$

Theorem 4 (Increase of the solution smoothness). Let the conditions of Theorem 2 hold, and let $\varphi_{1}^{-}$and $\varphi_{2}^{-}$be polynomials. Then for $\varphi_{1}^{+} \in \mathbf{E}_{\left(\varepsilon-\nu_{10}\right) h / 2}^{q-2}(\Omega), \varphi_{2}^{+} \in \mathbf{E}_{\left(\nu_{20}+\varepsilon\right) h / 2}^{q-2}(\Omega)$ a solution of the problem (1)-(3) exists in the space $\mathbf{E}_{\theta}^{2, q}(\overline{\mathcal{D}})$, where

$$
\theta(t)=\min \left\{\left(\varepsilon-\nu_{10}\right)(h / 2-t),\left(\nu_{20}+\varepsilon\right)(t-h / 2)\right\}
$$

If $\varphi_{1}^{+}$and $\varphi_{2}^{+}$are polynomials, then for $\varphi_{1}^{-} \in \mathbf{E}_{\left(\nu_{20}+\varepsilon\right) h / 2}^{q-2}(\Omega), \varphi_{2}^{-} \in \mathbf{E}_{\left(\varepsilon-\nu_{10}\right) h / 2}^{q-2}(\Omega)$, the solution of the problem (1)-(3) exists in the space $\mathbf{E}_{\theta}^{2, q}(\overline{\mathcal{D}})$, where

$$
\theta(t)=\min \left\{\left(\varepsilon-\nu_{10}\right)(t-h / 2),\left(\nu_{20}+\varepsilon\right)(h / 2-t)\right\}
$$

Proof. To estimate $u_{+}$and $u_{-}$one can use formulas (15) and (16). Further, the scheme of proof is analogical to the schemes of proofs of Theorems 2 and 3.

\section{REFERENCES}

1. R.P. Agarwal, I. Kiguradze, On multi-point boundary value problems for linear ordinary differential equations with singularities, Journ. Math. Anal. Appl., 297 (2004), 131-151. doi:10.1016/j.jmaa.2004.05.002

2. G. Akram, M. Tehseen, S. Siddiqi, Solution of a Linear Third order Multi-Point Boundary Value Problem using RKM, British Journ. of Math. \& Comp. Science, 3 (2013), 180-194. doi:10.9734/BJMCS/2013/2362.

3. I.I. Antypko, M.A. Perelman, On the uniqueness classes of the nonlocal multi-point boundary problem solutionsin the infinite layer, The function theory, funct. analysis and appl., 16 (1972), 98-109. (in Russian)

4. A.T. Assanova, On a solvability of a family of a multi-point boundary value problems for system of differential equations and the their application to the nonlocal boundary value problems, Math. Journ., Almaty, 13 (2013) no.3, 58-73.

5. A.T. Assanova, A.E. Imanchiev, On conditions of the solvability of nonlocal multi-point boundary value problems for quasi-linear systems of hyperbolic equations, Eurasian Math. Journ., 6 (2015), no.4, 19-28.

6. Banichuk NV, Ivanova SYu, Sharanyuk AV. Dynamics of Structures, Analysis and Optimization. Moscow: Nauka, 1989 (in Russian).

7. P. Bassanini, Iterative methods for quasilinear hyperbolic systems in the first canonic form, Appl. Anal., 12, (1981), no.2, 105-117. 
8. V.M. Borok, Uniqueness classes for the solution of a boundary problem with an infinite layer for systems of linear partial differential equations with constant coefficients, Math. USSR-Sb., 8 (1969), no.2, 275285.

9. V.M. Borok, M.A. Perel'man, On uniqueness classes for a solution of a boundary value problem in an infinite layer, Izv. Vyssh. Uchebn. Zaved., Ser. Math., 8, (1973), 29-34. (in Russian)

10. P. Cesari, A boundary value problem for quasilinear hyperbolic systems, Riv. math. Univ. Parma, 3 (1974), no.2, 107-131.

11. A. Dhamacharoen, K. Chompuvised, An efficient method for solving multipoint equation boundary value problems, Intern. Journ. of Math. and Comp. Sciences, 7 (2013), no.3, 329-333.

12. Yu.A. Dubinskii, A method of solution of partial differential equations, Dokl. Akad. Nauk SSSR, 258 (1981), no.4, 780-784.

13. Erofeev VI, Kazhaev VV, Semerikova NP. Waves in the rods. Dispersion. Dissipation. Nonlinearity. Moscow: Fizmatlit, 2002 (in Russian with an abstract in English).

14. L.V. Fardigola, Well-posed problems in a layer with differential operators in a boundary condition, Ukr. Mat. Zh., 44 (1992), no.8, 1083-1090. (in Russian)

15. L.V. Fardigola, Nonlocal two-point boundary-value problems in a layer with differential operators in a boundary condition, Ukr. Mat. Zh., 47 (1995), no.8, 1122-1128. (in Russian)

16. Filippov A.P. Oscillations of the deformable systems. Moscow: Mashinostroenie, 1970 (in Russian).

17. V.I. Gorbachuk, M.L. Gorbachuk, Boundary value problems for operator differential equations, Netherlands: Kluwer Academic Publishers, 1990.

18. J.R. Graef, L.Kong, Q. Kong, Higher order multi-point boundary value problems, Mathematische Nachrichten, 284 (2011), no.1, 39-52. doi:/10.1002/mana.200710179

19. R.J. Gu, K.L. Kuttler, M. Shillor, Frictional wear of a thermoelastic beam Journ. Math. Anal. And Appl., 242 (2000), 212-236.

20. C.P. Gupta, S.I. Trofimchuk, Solvability of a multi-point boundary value problem of Neumann type, Abstr. and Appl. Analysis, 4 (1999), no.2, 71-81.

21. R.A. Horn, Ch.R. Johnson, Matrix analysis, NY: Cambridge University Press, 2012.

22. V.S. Il'kiv, A multipoint nonlocal problem for partial differential equations, Diff. Uravn., 23 (1987), no.3, 487-492. (in Russian)

23. V.S. Il'kiv, Incorrect nonlocal boundary value problem for partial differential equations, In: Kadets V, Żelazko W (editors). North-Holland Math. Studies. Elsevier, 197 (2004), 115-121.

24. V.S. Il'kiv, Nytrebych Z.M., Estimate of the measure of level set for the solutions of differential equations with constant coefficients, Journ. of Math. Sciences. 217 (2016), no.2, 166-175. doi:10.1007/s10958-0162964-1

25. V.S. Il'kiv, Nytrebych Z.M., Pukach P.Y. Boundary-value problems with integral conditions for a system of Lame equations in the space of almost periodic functions, Electron. Journ. of Diff. Eq., 304, (2016), $1-12$.

26. V.S. Il'kiv, Nytrebych Z.M., Pukach P.Y., Nonlocal problem with moment conditions for hyperbolic equations, Electr Journ. of Diff. Eq., 265 (2017), 1-9.

27. V.S. Il'kiv, I.Ya. Savka, Nonlocal two-point problem for partial differential equations with linearly dependent coefficients, Journ. of Math. Sciences, 167 (2010), no.1, 47-61. doi:10.1007/s10958-010-9901-5

28. V.S. Il'kiv, N.I. Strap, Solvability of the nonlocal boundary-value problem for a system of differentialoperator equations in the Sobolev scale of spaces and in a refined scale, Ukrain. Math. Journ., 67 (2015), no.5, 690-710. doi:10.1007/s11253-015-1108-y

29. V.S. Il'kiv, N.I. Strap, Nonlocal boundary value problem for a differential-operator equation with nonlinear right part in a complex domain, Math. Stud., 45 (2016),no.2, 170-181. doi:10.15330/ms.45.2.170-181

30. V.S. Il'kiv, N.I. Strap, Nonlocal boundary-value problem for a differential-operator equation with weak nonlinearity in the spaces of Dirichlet-Taylor series with fixed spectrum, Journ. of Math. Sciences, 231 (2018), no.4, 572-585. doi:10.1007/s10958-018-3835-8

31. V.S. Il'kiv, N.I. Strap, Solvability of a nonlocal boundary-value problem for the operator-differential equation with weak nonlinearity in a refined scale of Sobolev spaces, Journ. of Math. Sciences, 218 (2016), no.1, 1-15. doi:10.1007/s10958-016-3006-8

32. T.S. Kagadiy, A.H. Shporta, The asymptotic method in problems of the linear and nonlinear elasticity theory, Naukovyi Visnyk Natsionalnoho Hirnychoho Univ., 3, (2015), 76-81.

33. P.I. Kalenyuk, Z.M. Nytrebych, On an operational method of solving initial-value problems for partial differential equations induced by generalized separation of variables, Journ. of Math. Sciences, 97 (1999), no.1, 3879-3887. 
34. I. Kiguradze, Some optimal conditions for the solvability of two-point singular boundary value problems, Funct. Diff. Equations, 10, (2003), 259-281.

35. L. Kong, Q. Kong, M.K. Kwong, J.S.W. Wong, Linear Sturm-Liouville problems with multi-point boundary conditions, Math. Nachricht, 286 (2013),no.11-12, 1167-1179. doi:10.1002/mana.201200187

36. R. Ma, D. O'Regan, Solvability of singular second order m-point boundary value problems, Journ. of Math. Analysis and Appl., 301 (2005), no.1, 124-134. doi:10.1016/j.jmaa.2004.07.009

37. Magrab EB. Vibrations of Elastic Systems with Applications to MEMS and NEMS. NY: Springer, 2012.

38. A.A. Makarov, On the existence of a well-posed two-point boundary value problem in a layer for systems of pseudo-differential equations, Differ. Uravn., 30 (1994), no.1, 144-150. (in Russian)

39. Z.M. Nitrebich, An operator method of solving the Cauchy problem for a homogeneous system of partial differential equations, J. Math. Sci., 81 (1996), no.6, 3034-3038.

40. J. Niu, Y.Zh. Lin, Chi.P. Zhang, Approximate solution of nonlinear multi-point boundary value problem on the half-line, Math. Modelling and Analysis, 17 (2012), no.2, 190-202. doi:10.3846/13926292.2012.660889

41. Z. Nytrebych, V. Il'kiv, P. Pukach, O. Malanchuk, On nontrivial solutions of homogeneous Dirichlet problem for partial differential equations in a layer, Krag. J. of Math., 42 (2018), no.2, 193-207.

42. Z. Nytrebych, O. Malanchuk, V. Il'kiv, P. Pukach, Homogeneous problem with two-point in time conditions for some equations of mathematical physics, Azerb. Jorn. of Math., 7 (2017), no.2, 174-190.

43. Z. Nytrebych, O. Malanchuk, V. Il'kiv, P. Pukach, On the solvability of two-point in time problem for PDE, Italian J. of Pure and Appl. Math., 38 (2017), 715-726.

44. M. Picone, Sui valori eccezionali di un parametro do cui dipende un equazione differentiale lineare ordinaria del secondo ordine, Pisa, 1909.

45. B.Yo. Ptashnyk, Problem of Vallee-Poussin type for hyperbolic equations with constant coefficients, Dopovidi Academii Nauk URSR, 10, (1966), 1254-1257. (in Ukrainian)

46. B.Yo. Ptashnyk, Problem of Vallee-Poussin type for linear hyperbolic equations with variable coefficients, Dopovidi Academii Nauk URSR, 2 (1967), 127-130. (in Ukrainian)

47. B.Yo. Ptashnyk, V.S. Il'kiv, I.Ya. Kmit', V.M. Polishchuk, Nonlocal boundary-value problems for partial differential equations. Kyiv: Naukova Dumka, 2002. (in Ukrainian)

48. B.Yo. Ptashnyk, M.M. Symotyuk, Multipoint problem with multiple nodes for partial differential equations, Ukr. Math. Journ., 55 (2003), no.3, 481-497.

49. P. Pucci, Problemi ai limiti per sistemi di equazioni iperboliche, Boll. Unione Mat. Ital. B, bf16 (1979), no.5, 187-99.

50. M.M. Symotyuk, I.R. Tymkiv, Problem with two-point conditions for parabolic equation of second order on time, Carpathian Math. Publ., 6 (2014), no.2, 351-359. (in Ukrainian) doi:10.15330/cmp.6.2.351-359

51. J.D. Tamarkin, On some general problems in the theory of ordinary linear differential equations and on the expansion in series of arbitrary functions, Petrograd, 1917 (in Russian).

52. M. Tatari, M. Dehgha, An efficient method for solving multi-point boundary value problems and applications in physics, Journ. of Vibr. and Contr., 18 (2011), no.8, 1116-1124. doi:10.1177/1077546311408467

53. Ch.J. Vallée-Poussin Sur l'équation différentielle linéaire du second ordre. Détermination d'une integrale par deux valeurs assignées. Extension aux equations d'ordre n, Journ. Math. de pura et appl., 9 (1929), no.8, 125-144.

Lviv Polytechnic National University

Lviv, Ukraine

ilkivv@i.ua

znytrebych@gmail.com

ppukach@gmail.com

mira.i.kopych@gmail.com

Received 05.02.2020

Revised 30.08.2020 